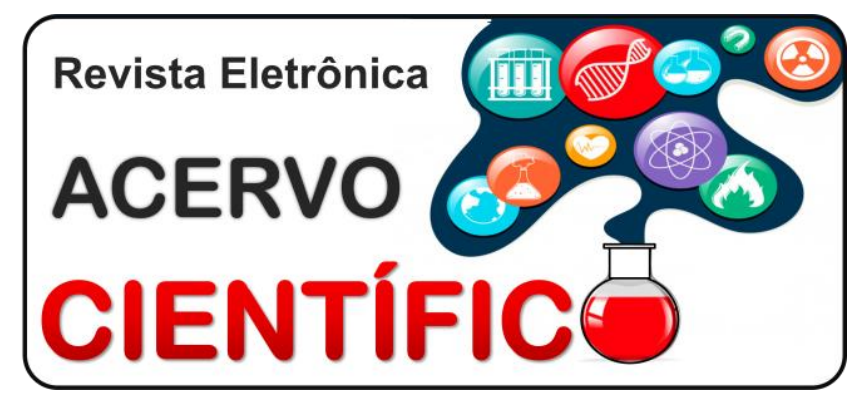

REVISÃO BIBLIOGRÁFICA

Recebido em: 7/2020

Aceito em: 8/2020

Publicado em: 10/2020

\title{
Reativação da doença de Chagas pós-transplante cardíaco
}

\author{
Reactivation of Chagas disease after cardiac transplantation
}

Reactivación de la enfermedad de Chagas tras transplante cardíaco

Brendon Arpini Rodrigues ${ }^{1 *}$, Humberto de Freitas Boy ${ }^{1}$, Abraão Albernaz Faria ${ }^{2}$, Ana Carolline Oliveira Torres², Caroline Silva de Araujo Lima ${ }^{3}$, Luciana Chagas Prado ${ }^{4}$, Luciano Ribeiro Coelho², Maria Angélica Otero de Melo dos Reis ${ }^{5}$, Maria Carolina Rodrigues Lopes ${ }^{1}$, Thaiz Geovana Bezerra6.

Resumo: Esse artigo buscou discutir a reativação da Doença de Chagas em pacientes que passaram por transplante cardíaco. Trata-se de uma revisão bibliográfica em que são analisadas as manifestações clínicas decorrentes da reativação, assim como o diagnóstico, monitoramento e tratamentos abordados na literatura estudada. Assim, avalia-se essa particularidade apresentada pelo paciente com cardiomiopatia chagásica e sua influência no prognóstico após o procedimento cirúrgico. Devido à inexistência de tratamento capaz de reverter a evolução da doença, além de seu prognóstico ruim e elevados índices de morbidade e mortalidade, o transplante cardíaco apresenta-se como uma opção terapêutica para o estágio final dessa manifestação crônica da Doença de Chagas. Os pacientes chagásicos que passam por transplante cardíaco, além de enfrentar as complicações inerentes ao procedimento, encaram a possibilidade de reativação da doença após a imunossupressão necessária para evitar a rejeição do aloenxerto. A reativação pode acontecer devido ao desequilíbrio na relação hospedeiro-parasita pela imunossupressão e/ou persistência dos protozoários no coração explantado. Finalmente, salienta-se a necessidade de mais estudos visando a definição de uma abordagem mais efetiva. Destacamos também a importância do acompanhamento médico no pós-transplante a fim de identificar a reativação precocemente, o que aumenta as chances de um melhor prognóstico.

Palavras-chave: Transplante de coração, Doença de Chagas, Reincidência.

Abstract: This article aims to discuss the reactivation of Chagas Disease in patients that underwent heart transplantation. This is a literature review in which the clinical manifestations of the reactivation are approached, as well as the diagnosis, monitoring and treatments debated in the literature studied. Therefore, it is assessed this particularity presented by the patient with chagasic cardiomyopathy and its influence on the prognosis after the surgical procedure. Due to the inexistence of treatment capable of reversing the evolution of the disease, as well as its poor prognosis and elevated rates of morbidity and mortality, the heart transplantation presents itself as a therapeutic option for the end stage of this chronic manifestation of Chagas Disease. The patients with Chagas Disease that undergo heart transplantation, besides facing complications inherent to the procedure, they encounter the possibility of reactivation of the disease after the immunosuppression necessary to avoid allograft rejection. The reactivation may happen because of an

\footnotetext{
${ }^{1}$ Centro Universitário de Caratinga (UNEC), Caratinga - MG. *E-mail: brendon-arpini@hotmail.com

${ }^{2}$ Centro Universitário do Planalto Central Apparecido Dos Santos (UNICEPLAC), Brasília - DF.

${ }^{3}$ Faculdade Dinâmica Vale do Piranga (FADIP), Ponte Nova - MG.

${ }_{4}^{4}$ Centro Universitário de Volta Redonda (UniFOA), Volta Redonda - RJ.

5 Universidad Nacional de Rosario (UNR), Rosario - SF - Argentina.

${ }^{6}$ Universidade do Oeste Paulista (UNOESTE), Jaú - SP.
} 
unbalance on the host-parasite relationship due to the immunosuppression and/or persistence of the protozoa in the explanted heart. Finally, it is emphasized the urge for more studies aiming at ascertain a more effective approach. We enhance the importance of the medical follow-up post-transplant with the purpose of precociously identify reactivation, which raises the chances of a better prognosis.

Keywords: Heart transplantation, Chagas disease, Recidivism.

Resumen: Este artículo buscaba discutir la reactivación de la enfermedad de Chagas en pacientes que se sometieron a un trasplante de corazón. Se trata de una revisión bibliográfica en la que se analizan las manifestaciones clínicas resultantes de la reactivación, así como el diagnóstico, el seguimiento y el tratamientos abordados en la literatura estudiada. Así, se evalúa esta particularidad que presenta el paciente con miocardiopatía de Chagas y su influencia en el pronóstico después del procedimiento quirúrgico. Debido a la inexistencia de un tratamiento capaz de revertir la evolución de la enfermedad, así como a su mal pronóstico y a las altas tasas de morbilidad y mortalidad, el trasplante de corazón se presenta como una opción terapéutica para la etapa final de esta manifestación crónica de la enfermedad de Chagas. Los pacientes con enfermedad de Chagas que se someten a un trasplante de corazón, además de enfrentarse a las complicaciones inherentes al procedimiento, se enfrentan a la posibilidad de reactivación de la enfermedad tras la inmunosupresión necesaria para evitar el rechazo del injerto. La reactivación puede ocurrir debido al desequilibrio en la relación huésped-parásito por la inmunosupresión y/o la persistencia de protozoos en el corazón explantado. Por último, se destaca la necesidad de realizar más estudios para definir un enfoque más eficaz. También destacamos la importancia del seguimiento médico posterior al trasplante para identificar la reactivación temprana, lo que aumenta las posibilidades de un mejor pronóstico.

Palabras clave: Trasplante de corazón, Enfermedad de Chagas, Reincidencia.

\section{INTRODUÇÃO}

A Doença de Chagas, causada pelo protozoário Trypanosoma cruzi, é uma zoonose endêmica na América Latina, que pode ser transmitida ao homem pelas vias vetoriais, transfusional, congênita, acidental, oral e por transplantes de órgãos. Nos últimos anos, a prevalência da infecção humana é estimada pela Organização Mundial da Saúde (OMS) em 16 a 18 milhões de pessoas infectadas na América latina, considerando que no Brasil 5 a 7 milhões de chagásicos, sendo que aproximadamente $30 \%$ dos indivíduos infectados desenvolvem a forma crônica da doença (ALMEIDA DR, 2016).

Na doença há duas fases características: fase aguda e fase crônica. Na fase aguda há a alta parasitemia associada à infestação e multiplicação do parasita. Se a contaminação tiver ocorrido por via oral, como pela ingestão de caldo de cana ou açaí contaminado com $T$. cruzi, maior a carga parasitária, tendo um prognóstico pior em comparação às outras vias de transmissão, que cursam com a forma benigna na maioria das vezes (SIMÕES MV, et al., 2018).

Quando há a remissão da fase aguda o paciente entra na fase crônica, passando pelo período indeterminado, onde apresenta positividade sorológica para a doença. Nesse estágio, pode haver sinais leves de comprometimento cardíaco, mas sem sintomas específicos. Após ocorrido a cronificação de sintomas cardíacos com o dano de fibras miocárdicas pelo protozoário e aumento de fibrose reparativa, o hospedeiro entra em um quadro de miocardiopatia dilatada, acompanhada de arritmias graves, insuficiência cardíaca refratária, disfunção ventricular e complicações tromboembólicas, podendo ou não acompanhar de congestão pulmonar (SIMÕES MV, et al., 2018; ALMEIDA DR, 2016).

Das complicações da doença de Chagas, a miocardiopatia dilatada é a mais perigosa, sendo responsável por alterações como no ritmo sinusal e na ejeção ventricular. Por isso, o transplante de coração (TC) é considerado padrão-ouro para a terapêutica de pacientes com essa insuficiência cardíaca refratária, já que o paciente apresenta grande limitação funcional e alta mortalidade. Apesar disso, não são todos os pacientes que podem ser candidatos ao TC. Pacientes com hipertensão pulmonar, doenças vasculares periféricas graves, incompatibilidade $A B O$ (O sistema $A B O$ classifica grupos sanguíneos em tipos $A, B, A B$ e O), doença psíquica grave e dependência química são algumas das contra indicações avaliadas antes de 
submeter o paciente ao TC (MANGINI S, 2015). A indicação é, portanto, feita a partir de uma estratificação de riscos dos pacientes com Cardiopatia chagásica crônica New York Heart Association (NYHA), sendo as classes III e IV mais indicadas para o transplante (SIMÕES MV, et al., 2017; DINARDI LFL, et al., 2012; MANGINI S, et al., 2015).

Além disso, com o uso de imunossupressores para evitar uma possível rejeição ao órgão transplantado, há uma grande chance de reativação da doença de Chagas, situação que necessita de cuidados especiais do médico para com o paciente, analisando possíveis sinais e sintomas de reativação (MANGINI S, et al., 2015; DINARDI LFL, et al., 2012).

Neste estudo, pretende-se analisar os achados relevantes que a literatura traz, nos últimos dez anos, sobre a reativação da doença de Chagas após o transplante cardíaco e a necessidade de acompanhamento do paciente pós-transplantado pelo médico para avaliar possíveis sinais e sintomas dessa reativação.

\section{REVISÃO BIBLIOGRÁFICA}

A doença de Chagas, em um terço dos indivíduos, se desenvolve em sua forma crônica, acometendo o coração e / ou trato gastrointestinal, anos após a fase aguda (BENATTI RD, et al., 2017). A forma crônica cardíaca é a mais grave, acometendo cerca de 30 a $40 \%$ de todos os indivíduos infectados com Chagas (ALMEIDA DR, 2016; BENVENUTI LA, et al., 2014). Esta tem como manifestação a cardiomiopatia chagásica que se caracteriza como uma doença evolutiva, de prognóstico severo, com índices altos de morbidade e mortalidade os quais alcançam cerca de $30 \%$. Assim, por meio de estudos recentes foi detectado que esses pacientes apresentam menor sobrevida. Isso se deve a suas características principais de disfunção e arritmias de ambos os ventrículos, além de complicações tromboembólicas (BENCK L, et al., 2018; BENVENUTI LA, et al., 2014; SIMÕES MV, et al., 2018).

O TC é a única forma de tratamento para pacientes com cardiomiopatia em estágio terminal, sendo essa classificação feita de acordo com a NYHA a qual analisa a capacidade funcional cardíaca (BESTETTI RB, 2016; DINARDI LFL, et al., 2012; PERADEJORDI ML, et al., 2016). Além disso, pacientes com insuficiência cardíaca decorrentes da doença de Chagas apresentam um pior prognóstico do que pacientes não chagásicos, com taxas de mortalidade de $50-60 \%$ em dois anos, indicando a extrema necessidade de avaliação para uma possível intervenção cirúrgica (ALMEIDA DR, 2016). O risco de morte para pacientes com a forma crônica cardíaca da doença de Chagas, pode ser calculada por meio do Escore de Rassi, reforçando a ideia anterior da melhor seleção e necessidade de transplante cardíaco (SIMÕES MV, et al., 2018).

Porém, por ser uma doença infecciosa sistêmica incurável, com influência de ativação imunológica, existe um potencial de reativação da infecção devido ao tratamento por imunossupressão típico póstransplante, o que pode comprometer a funcionalidade do aloenxerto a curto e a longo prazo (ALMEIDA DR, 2016; BURGOS JM, et al., 2010; GRAY EB, et al., 2018). Dessa forma, de acordo com o estudo de Melo CF, et al. (2020) existem algumas contraindicações absolutas para realização de transplantes cardíacos, sendo uma delas pacientes que possuem restrição medicamentosa do uso de imunossupressores. Portanto, uma vez que indivíduos infectados por Chagas possuem essa restrição, inicialmente houve a contraindicação do TC nessas condições, mas com a evolução de alguns estudos, comprovou-se a eficácia nesse procedimento (KRANSDORF EP, et al., 2013). Comprovando-se tais fatos, apesar das taxas de reativação serem altas, a mortalidade é baixa, em cerca de $0,9 \%$, fortalecendo a ideia de que com monitoramento e tratamento adequado há uma melhora substancial do prognóstico (BENATTI RD, et al., 2017; BACAL F, et al., 2010; BENVENUTI LA, et al., 2014; VIERA JL, et al., 2019; MANGINI S, et al., 2015).

Em relação a epidemiologia tem-se que a região endêmica da Doença de Chagas está localizada na América Central e do Sul que apresenta um total aproximado de 8 milhões de pessoas infectadas pelo Trypanosoma cruzi (BENATTI RD, et al., 2017). No âmbito brasileiro, esse número encontra-se ainda mais relevante, visto que apresenta entre 2,5 a 5 milhões de indivíduos infectados presentes em todos os seus estados (ALMEIDA DR, 2016). 
Ademais, devido ao processo imigratório de latino-americanos, se tem uma presença elevada de indivíduos infectados fora da sua região endêmica, totalizando cerca de 400.000 habitantes com Doença de Chagas nos Estados Unidos e em países da Europa (BENATTI RD, et al., 2018; VIERA JL, et al., 2019). Dessa forma, justifica-se o número elevado de TCs em pacientes com cardiomiopatia Chagásica na América (KRANSDORF EP, et al., 2014; BENATTI RD, et al., 2018). Logo, a cardiomiopatia chagásica apresenta $35 \%$ das indicações para TC no Brasil. Sendo a terceira posição líder de transplante atualmente no país, pela sua possibilidade de resolução da fase terminal da doença (KRANSDORF EP, et al., 2014; FIORELLI Al,et al., 2011).

Segundo o estudo de Fiorelli Al, et al. (2011), o TC pode apresentar algumas complicações no pósoperatório que requerem cuidados, pois podem levar a óbito. Dentre as principais evoluções desfavoráveis desse procedimento temos as infecções, a rejeição, as neoplasias e, principalmente, a reativação da doença de Chagas, devido ao processo de imunossupressão pós-transplante. Com isso, as taxas de reativação são significativas em transplantes realizados na América Latina, variando de $20 \%$ a 90\% (GRAY EB, et al., 2018; VIERA JL, et al., 2019).

\section{Casuísticas}

O tratamento medicamentoso por meio da imunossupressão após o transplante é essencial para evitar a rejeição do aloenxerto. Porém, nos pacientes portadores de Chagas, a imunossupressão pode causar a reativação da doença no organismo (NOGUEIRA SS, et al., 2018; MANGINI S, et al., 2015). Dessa forma, acredita-se que a reativação acontece devido ao desequilíbrio da relação entre hospedeiro e parasita, podendo estar associada não só a decadência do sistema imune, como também pela proliferação e disseminação do parasita, o qual pode estar, supostamente, em reservatórios corporais (BENVENUTI LA, et al., 2014).

Além disso, achados como a persistência dos parasitas nos corações explantados e a gravidade da miocardite, possuem relação com a proporção da reativação. Ademais, também se levanta a hipótese de que há uma ligação da incidência da reativação e a técnica utilizada no transplante cardíaco, sendo que em transplantes bicavais, a reativação se torna menos comum do que em transplantes biatriais (BURGOS JM, et al., 2010). Contudo, há a necessidade de mais pesquisas para avançar na descoberta de fatores do hospedeiro determinantes para reativação da doença mesmo após o transplante (KRANSDORF EP, et al., 2014).

Deve-se ressaltar que no primeiro ano pós-transplante o uso de imunossupressores é mais intenso, assim, a reativação da doença de Chagas se torna mais propensa, e, visto que esta, reincide na sua forma aguda, o tratamento se torna mais eficiente. Porém é indispensável que nesse período haja maior monitoramento do paciente, para evitar piores consequências, por meio da identificação e tratamento da reativação o quanto antes (BACAL $F$, et al., 2010; DINARDI LFL, et al., 2012; BENCK L, et al., 2018; BENVENUTI LA, et al., 2014; MANGINI S, et al., 2015).

\section{Manifestações Clínicas}

As manifestações clínicas de infecções recidivas pelo $\mathrm{T}$. cruzi apresentam um quadro clínico com sintomas que podem estar relacionados ao coração e/ou outros órgãos e sistemas (BENATTI RD, et al., 2017; FIORELLI Al, et al., 2011; MANGINI S, et al., 2015). Podem ser evidenciados achados como lesões cutâneas (chagomas e nódulos) e subcutâneas (paniculite inflamatória), acometimentos neurológicos do Sistema Nervoso Central (meningoencefalite) e medular, também podendo apresentar febre, como um sintoma sistêmico (BENATTI RD, et al., 2017; FIORELLI Al, et al., 2011; GRAY EB, et al., 2018; MANGINI S, et al., 2015).

Ao analisar os sintomas associados ao aloenxerto submetido à reativação, pode ser perceptível sinais e sintomas de insuficiência cardíaca devido ao retorno da miocardite, a qual pode ser silenciosa ou se manifestar de forma grave como o choque cardiogênico e/ou como o bloqueio atrioventricular resultando na disfunção ventricular grave (BENATTI RD, et al., 2017; BENCK L, et al., 2018; GRAY EB, et al., 2018; MANGINI S, et al., 2015). Contudo, tais acometimentos citados podem se assemelhar ao processo de 
rejeição aguda do aloenxerto, o que torna mandatório o monitoramento do processo de reativação (BENATTI RD, et al.,2017).

\section{Diagnóstico e monitoramento}

Para evidenciar de maneira antecipada a reativação do processo infeccioso chagásico no receptor após o transplante, faz-se necessário o monitoramento clínico e laboratorial seguindo um protocolo bem estruturado para o êxito do transplante (VIERA JL, et al., 2019). Contudo, apesar de bem documentado esse tipo de protocolo, ainda não existe um consenso de abordagem padrão, visto que, os estudos analisados apresentam algumas divergências na conduta realizada (GRAY EB, et al., 2018).

A análise pós transplante deve iniciar por meio do exame clínico, seguido por exames de imagem e laboratoriais (BESTETTI RB, 2016; KRANSDORF EP, et al., 2014; KRANSDORF EP, et al.,2013; GRAY EB, et al., 2018). A frequência de análise do receptor após o transplante se faz necessária para um acompanhamento minucioso em relação a reativação da doença de Chagas (Tabela 1).

Tabela 1 - Frequência de análise do receptor segundo o tempo após o transplante cardíaco, para o monitoramento da reativação da Doença de Chagas.

\begin{tabular}{ll}
\hline Tempo pós-transplante & Frequência de análise \\
\hline $1^{\circ}$ e $2^{\circ}$ mês & Semanal \\
\hline $3^{\circ}$ ao $6^{\circ}$ mês & A cada 2 semanas \\
\hline $6^{\circ}$ ao $12^{\circ}$ mês & Por mês \\
\hline $13^{\circ}$ ao $24^{\circ}$ mês & A cada 3 meses \\
\hline 2 anos a diante & A cada 6 meses
\end{tabular}

Fonte: RODRIGUES BA, et al., 2020. Adaptado de KRANSDORF EP, et al., 2014.

Para a análise de disfunção do aloenxerto deve-se utilizar de eletrocardiograma (ECG) e ecocardiograma. O ECG possibilita a identificação de bloqueios de condução e o ecocardiograma para análise de disfunções ventriculares reincidentes (BENATTI RD, et al., 2017; KRANSDORF EP, et al., 2013).

Dentre os estudos laboratoriais que podem ser realizados o mais indicado é a Reação em Cadeia da Polimerase (PCR) que consiste em uma técnica de análise do ácido desoxirribonucléico (DNA) do T. cruzi (BENVENUTI LA, et al., 2014; PERADEJORDI ML, et al., 2016). A reativação da Doença de Chagas no paciente após o transplante se caracteriza pela presença do parasita na corrente sanguínea em sua forma tripomastigota, comum a forma aguda da doença, dessa forma, possibilitando que a PCR seja o método mais sensível para detecção do parasita de forma mais precoce, dias a semanas antes das manifestações clínicas da reativação (MACHADO FS, et al. 2012; GRAY EB, et al., 2018). Porém, apesar de seus benefícios, o acesso PCR é restrito a laboratórios especializados (NOGUEIRA SS, et al. 2018).

Após as manifestações clínicas, outra forma de detecção da reativação, acontece por meio da realização da biópsia, com técnicas histopatológicas e imuno-histoquímicas, do tecido suspeito, como do endomiocárdico e/ou cutâneo para detecção da forma amastigota do parasita (BACAL F, et al., 2010; MANGINI S, et al., 2015; VIERA JL, et al., 2019; GRAY EB, et al., 2018). Além disso, exames como xenodiagnóstico e hemoculturas podem ser utilizados para o diagnóstico, porém nem todos os estudos indicam sua utilização devido a possibilidade de falsos positivos (ALMEIDA DR, 2016; BACAL F, et al., 2010; DINARDI LFL, et al., 2012).

Em exames que buscam a presença de tripomastigotas, pode-se analisar o sangue por meio de exames de microscopia de coletas frescas de Buffy Coat ou Giemsa do sangue periférico e, também, por meio da análise do esfregaço do líquido cefalorraquidiano (KRANSDORF EP, et al., 2014; BENATTI RD, et al., 2017; GRAY EB, et al., 2018). Ademais, imunodiagnósticos podem ser realizados por meio de técnicas como ELISA, hemaglutinação indireta (IHA) e imunofluorescência indireta (IIF). Devido à alta efetividade do IIF e ELISA, estes são os testes indicados para a substituição do PCR, em vista do seu difícil acesso (NOGUEIRA SS, et al. 2018). 
O protocolo de monitoramento não evita necessariamente a reativação da doença, entretanto permite a identificação precoce, viabilizando tratamentos imediatos os quais podem evitar resultados mais severos (GRAY EB, et al., 2018).

\section{Imunossupressão}

O esquema de imunossupressão típico pós-transplante cardíaco prioriza a prevenção da rejeição do aloenxerto, mas pode ocasionar um desequilíbrio na relação hospedeiro-parasita em pacientes portadores crônicos da doença de Chagas (FIORELLI Al, et al., 2011; BENVENUTI LA, et al., 2014; MANGINI S, et al., 2015). Na maioria dos estudos analisados foi ressaltada a necessidade de alteração da imunossupressão típica no pós-operatório de pacientes submetidos ao TC por motivo de cardiomiopatia Chagásica, no qual se tem a adaptação do medicamento e da dosagem (ALMEIDA DR, 2016; FIORELLI Al, et al., 2011; BACAL F, et al., 2010; MACHADO FS, et al. 2012; MANGINI S, et al., 2015).

$O$ uso do medicamento micofenolato mofetil (MMF) tem sido correlacionado com maiores taxas de reativação da doença de Chagas, em relação a Azatioprina (BACAL F, et al., 2010; BENATTI RD, et al., 2018; VIERA JL, et al., 2019; MANGINI S, et al., 2015). Segundo Fiorelli Al, et al. (2011) e Dinardi LFL, et al. (2012) o protocolo inicial de imunossupressão deve restringir-se ao uso de ciclosporina, corticóide e azatioprina. O protocolo de transplante cardíaco da UNIFESP demonstra um comparativo entre as doses utilizadas no pós-operatório de pacientes chagásicos e não chagásicos, o que comprova a necessidade da redução de doses de imunossupressores e do seu tempo de utilização em pacientes portadores de Chagas. Além disso, o corticoide foi suspenso antecipadamente ao final do segundo mês (Tabela 2). No entanto, é importante ressaltar que muitos autores consideram que o uso de corticoides é um agravante importante para a reativação da doença e recomendam a sua retirada do protocolo de imunossupressão, podendo contribuir, também, na redução do aparecimento de neoplasias (FIORELLI Al, et al., 2011; DINARDI LFL, et al., 2012).

Tabela 2 - Comparativo de doses medicamentosas, utilizadas após o transplante cardíaco, entre paciente Chagásicos e não-Chagásicos.

\begin{tabular}{ccc}
\hline Droga utilizada & Pacientes Chagásicos & Pacientes não Chagásicos \\
\hline Metilprednisolona & $500 \mathrm{mg}$ & $750-1000 \mathrm{mg}$ \\
\hline \multirow{2}{*}{ Prednisona } & $0,5 \mathrm{mg} / \mathrm{kg} / \mathrm{dia}$ & $1 \mathrm{mg} / \mathrm{kg} / \mathrm{dia}-$ Até 30 dias \\
& Até o $2^{\circ} \mathrm{mês}$ & $0,1-0,2 \mathrm{mg} / \mathrm{kg} / \mathrm{dia}-$ Após 30 dias \\
\hline Azatioprina & $1-2 \mathrm{mg} / \mathrm{kg} / \mathrm{dia}$ & $2-2,5 \mathrm{mg} / \mathrm{kg} / \mathrm{dia}$ \\
\hline Ciclosporiza & $3,5 \mathrm{mg} / \mathrm{kg} / \mathrm{dia}$ & $5 \mathrm{mg} / \mathrm{kg} / \mathrm{dia}$
\end{tabular}

Fonte: RODRIGUES BA, et al., 2020. Adaptado de ALMEIDA DR, 2016.

\section{Tratamento}

Identificada a reativação, inicia-se o tratamento com antiparasitário concomitante a diminuição da imunossupressão (ALMEIDA DR, 2016). Existem três possíveis medicamentos para tratamento da reativação: benzonidazol, nifurtimox e allopurinol (FIORELLI AI, et al., 2011; GRAY EB, et al., 2018; VIERA JL, et al., 2019; MANGINI S, et al., 2015). A dosagem recomendada para adultos do benzonidazol varia entre 5 a $10 \mathrm{mg} / \mathrm{kg} /$ dia durante o período de 60 dias, do allopurinol varia entre 600 a $900 \mathrm{mg} / \mathrm{dia}$ por 60 dias, enquanto o do nifurtimox varia entre 8 a $10 \mathrm{mg} / \mathrm{kg} /$ dia durante 60 a 90 dias (ALMEIDA DR, 2016; FIORELLI Al, et al., 2011; BACAL F, et al., 2010; DINARDI LFL, et al., 2012; GRAY EB, et al., 2018; VIERA JL, et al., 2019).

A terapia induzida pelo benzonidazol é capaz de extinguir os parasitas circulantes em até 2 semanas e seus efeitos colaterais são possivelmente amenizados pela imunossupressão (FIORELLI Al, et al., 2011). Embora possa apresentar efeitos colaterais como dermatite alérgica, parestesia e anorexia o benzonidazol segue como o medicamento mais indicado para o tratamento da reativação (ALMEIDA DR, 2016; KRANSDORF EP, et al., 2014; BENATTI RD, et al., 2017; MACHADO FS, et al., 2012; PERADEJORDI ML, et al., 2016). 
O tratamento profilático com antitrypanossômicos é um procedimento controverso, mas majoritariamente é contraindicado devido aos seus efeitos colaterais, além do fato de que os pacientes cronicamente infectados ainda podem apresentar a reativação da doença, pois esse tratamento não tem o potencial de erradicar a doença, necessitando do monitoramento do paciente (KRANSDORF EP, et al., 2014; GRAY EB, et al., 2018; MACHADO FS, et al. 2012).

\section{CONSIDERAÇÕES FINAIS}

Por meio da análise bibliográfica desse estudo, pode-se concluir que o transplante cardíaco é o tratamento de referência em pacientes portadores de cardiomiopatia chagásica com insuficiência cardíaca, conforme indicação clínica. A reativação da doença é a principal complicação relacionada com a imunossupressão induzida para impedir a rejeição do aloenxerto. Além disso, é importante salientar não só a necessidade de mais estudos na área em busca de melhores abordagens, mas também o imprescindível acompanhamento médico a fim de reduzir casos de complicações pela reativação da doença e aumentar o sucesso do transplante.

\section{REFERÊNCIAS}

1. ALMEIDA DR. Transplante cardíaco na Doença de Chagas. Rev. Soc. Cardiol. Estado de São Paulo, 2016; 26(4): 266-71.

2. BACAL F, et al. Transplantation for Chagas' Disease: An Overview of Immunosuppression and Reactivation in the Last Two Decades. Clinical Transplantation, 2010; 24(2): 29-34.

3. BENATTI RD, et al. Heart Transplant Outcomes in Patients with Chagas Cardiomyopathy in the United States. Clinical Transplantation, 2018; 32(6): e13279.

4. BENATTI RD, et al. Heart Transplantation for Chagas Cardiomyopathy. The Journal of Heart and Lung Transplantation, 2017; 36(6):597-603.

5. BENCK L, et al. Diagnosis and Management of Chagas Cardiomyopathy in the United States. Current Cardiology Reports, 2018; 20(12): 1-8.

6. BENVENUTI LA, et al. Trypanosoma Cruzi Persistence in the Native Heart Is Associated with High-Grade Myocarditis, but Not with Chagas 'Disease Reactivation after Heart Transplantation. The Journal of Heart and Lung Transplantation: The Official Publication of the International Society for Heart Transplantation, 2014; 33(7): 698 703.

7. BESTETTI RB. Cardiomiopatia Chagásica crônica - diagnóstico e tratamento. Rev. Soc. Cardiol. Estado de São Paulo, 2016; 26(4): 246 - 252.

8. BURGOS JM, et al. Molecular Identification of Trypanosoma Cruzi Discrete Typing Units in End-Stage Chronic Chagas Heart Disease and Reactivation after Heart Transplantation. Clinical Infectious Diseases: An Official Publication of the Infectious Diseases Society of America. 2010; 51(5): 485 - 495.

9. DINARDI LFL, et al. Transplante cardíaco na doença de Chagas. Rev Med, São Paulo, 2012; 91(4): $229-240$.

10. FIORELLI Al, et al. Heart Transplantation in 107 Cases of Chagas Disease. Transplantation Proceedings, 2011; 43(1): 220-24.

11. GRAY EB, et al. Reactivation of Chagas Disease among Heart Transplant Recipients in the United States $2012-$ 2016. Transplant Infectious Disease: An Official Journal of the Transplantation Society. 2018; 20(6): $1-24$

12. KRANSDORF EP, et al. Chagas Disease in Solid Organ and Heart Transplantation. Current Opinion in Infectious Diseases, 2014; 27(5): 418-4124.

13. KRANSDORF EP, et al. Heart Transplantation for Chagas Cardiomyopathy in the United States. Am J Transplant, 2013; 13(12): 3262-3268.

14. MACHADO FS, et al. Chagas Heart Disease: Reporton Recent Developments. Cardiology in Review. $2012 ; 20$ (2): $53-65$.

15. MANGINI S, et al. Transplante cardíaco: revisão. Einstein, São Paulo, 2015; 13(2): 310 - 318.

16. MELO CF, et al. "Entre el pulsar y el morir: la vivencia de pacientes que esperan el trasplante cardíaco". Enferm. glob, 2020; (58): $351-363$.

17. NOGUEIRA SS, et al. Challenges of Immunosuppressive and Antitrypanosomal Drug Therapy after Heart Transplantation in Patients with Chronic Chagas Disease: A Systematic Review of Clinical Recommendations. Transplantation Reviews, 2018; 32 (3):157- 167.

18. PERADEJORDI ML, et al. "Resultados del trasplante cardíaco según el perfil clínico del receptor: 21 años de experiencia". Rev. argent. cardiol, 2016; 243-249.

19. SIMÕES MV, et al. Chagas Disease Cardiomyopathy. International Journal of Cardiovascular Sciences, 2018; 31(2): 173-189.

20. VIERA JL, et al. Chagas Cardiomyopathy in Latin America Review. Curr Cardiol Rep., 2019; 21(2): 1- 8. 